\title{
Administración, ambiente organizacional y satisfacción en el trabajo: fundamentos filosóficos, conceptuales y sociológicos
}

\author{
Elena A. Martínez Ibarra \\ Recibido: 12 de febrero de 2013 \\ Universidad de Puerto Rico, Río Piedras \\ Aceptado: 3 de mayo de 2013
}

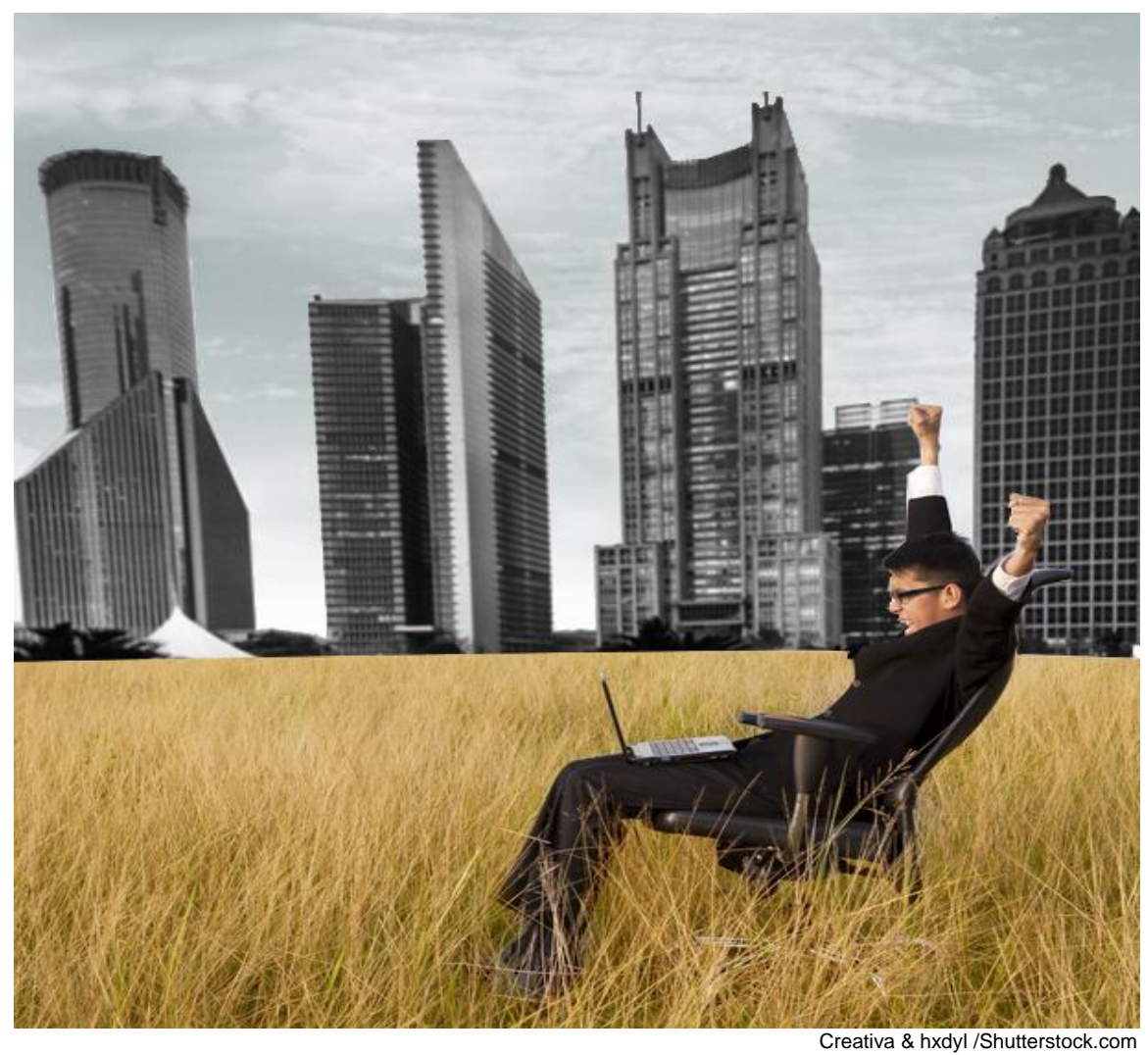

\section{RESUMEN}

Este trabajo es un recorrido filosófico, conceptual y sociológico del concepto de administración y de otros temas relacionados con la psicología industrial, al ambiente organizacional y la satisfacción en el trabajo. Se comienza con la influencia de los grandes filósofos como Sócrates y Platón y se continúa con eventos significativos como la Revolución Industrial y el efecto de la misma en el desarrollo de nuevos estilos y estrategias gerenciales. Se enfatiza la psicología industrial organizacional porque uno de sus retos es lograr la armonía en el trabajo; y los últimos años se han destacado por la búsqueda de nuevas estructuras y sistemas que garanticen el mejor funcionamiento de las organizaciones. También se discute el concepto de ambiente organizacional porque está directamente relacionado con el comportamiento organizacional, la gerencia de recursos humanos, a la psicología industrial organizacional y al comportamiento humano en la organización.

\section{PALABRAS CLAVES}

Administración, psicología industrial organizacional, conducta organizacional, ambiente organizacional, satisfacción en el trabajo 


\begin{abstract}
The present is a philosophical, sociologic, and conceptual journey of the notion of administration, and other concepts related to Industrial Psychology like organizational environment, and satisfaction in the workplace. It starts with the influence of the great philosophers like Socrates and Plato. Later, it continues with the effects of relevant events such as the Industrial Revolution on the development of new styles and management strategies. This research empathizes on Industrial and Organizational Psychology since late findings are identified with the search of structures, systems, and mechanisms oriented to guarantee the best performance of the organizations. One of the challenges of this psychology aims to create consensus in the work place. Likewise, the concept of organizational environment is discussed since it is directly related to organizational behavior, human resources management, industrial psychology, and human behavior in the organization.
\end{abstract}

\section{KEY WORDS}

Administration, industrial organizational psychology, organizational behavior, organizational environment, work satisfaction.

\section{Introducción}

a crisis económica mundial, que cada día nos afecta más, reduce las oportunidades de trabajo para los diferentes sectores de la sociedad, aumenta el desempleo y deteriora las condiciones en el lugar de trabajo, ya que al aumentar los problemas de las personas aumentan los niveles de stress en la organización y la insatisfacción en el trabajo.

Este ambiente dentro de la organización y la satisfacción en el trabajo son dos de las áreas más importantes con las que deben trabajar los especialistas en administración y conducta de los empleados dentro de una organización. En este trabajo se realiza una revisión amplia de la literatura relacionada a la administración dentro de la empresa. Se expone que tras esa administración que tiene que considerar y manejar tantos problemas, hay cientos de años de esfuerzos, de situaciones y de personas que influenciaron en su desarrollo.

El trabajo comienza con una revisión histórica que incluye la influencia de grandes filósofos como Platón y Sócrates. Eventualmente se incorporan otros movimientos e influencias como la filosofía moderna y la revolución industrial. Después se incluyen las teorías de grandes pensadores y psicólogos industriales de la actualidad como Muchinsky y Chiavenato; y algunos estudiosos puertorriqueños como el Dr. Carlos Toro y el Dr. Ángel Rivera que se dedica a estudiar estos temas en las organizaciones del país.

En el análisis de otros expertos se puede, además, apreciar el origen de nuevas disciplinas relacionadas a la administración como la psicología industrial, el ambiente dentro de y el desarrollo organizacional y el ambiente en el trabajo.

En la parte final de este trabajo se toca el caso de Puerto Rico y se llegan a conclusiones sobre lo que es el ambiente organizacional y la necesidad de realizar más investigaciones relacionadas a ese ambiente y a la satisfacción en el trabajo.

\section{Origen y desarrollo de la Administración}

La administración que conocemos hoy es el resultado histórico e integrado de la contribución de numerosos pioneros: filósofos, físicos, economistas, estadistas e incluso empresarios que con el transcurso del tiempo desarrollaron y divulgaron obras y teorías en su campo de actividades. No es de extrañar, entonces, que la administración moderna utilice conceptos y principios descubiertos y usados en las ciencias matemáticas, humanas (psicología, sociología, biología, educación, etc.), físicas 
(física, química, etc.), así como en el derecho, la ingeniería, etc. (Chiavenato, 2000).

Desde la antigüedad, la administración ha recibido gran influencia de la filosofía. El filósofo griego Sócrates, quien vivió del año 470 al 399 a.C., en su discusión con Nicómano, visualiza la administración como una habilidad personal separada del conocimiento técnico y de la experiencia. También Platón, el filósofo griego discípulo de Sócrates, quien vivió del 429 al 347 a.C., se preocupó por los problemas políticos y sociales inherentes al desarrollo social y cultural del pueblo griego; y en su libro La república expuso su punto de vista sobre el estilo democrático de gobierno y cómo debe ser la administración de los negocios públicos. Por otro lado, Aristóteles, quien vivió del 384 al 322 a.C., abrió nuevos horizontes al conocimiento humano de la época y fue el creador de la lógica. En su libro Política, estudió la organización del estado y distinguió las tres formas de administración pública: monarquía o gobierno de una sola persona (que puede acabar con la tiranía); aristocracia o gobierno de una elite (que puede generar en oligarquía); democracia o gobierno del pueblo (que puede convertirse en anarquía).

Entre la antigüedad y el inicio de la edad moderna, la filosofía estudió gran variedad de temas ajenos a los asuntos administrativos. Solo a partir de Francis Bacon (1561-1626), filósofo y estadista inglés, considerado el fundador de la lógica moderna, se encuentra alguna preocupación por separar experimentalmente lo esencial de lo accidental. Bacon se anticipó a uno de los principios básicos de la administración conocido como principio de la prevalencia de lo principal sobre lo accesorio (Chiavenato, 2000). No obstante, el mayor exponente de la obra fue Rene Descartes (1596-1650), filósofo, matemático y físico francés, considerado el fundador de la filosofía moderna. Descartes creó las famosas coordenadas cartesianas e impulsó la matemática y la geometría de la época. Los principios cartesianos contienen algunos de los principios de la administración moderna: división del trabajo, orden y control, entre otros.

Con el surgimiento de la filosofía moderna la administración dejó de recibir influencias filosóficas, puesto que el objeto de estudio de la filosofía se aleja de los asuntos organizacionales (Chiavenato, 2000). La administración, sin embargo, sigue evolucionando por los eventos que ocurren a través de la historia, por ejemplo, la Revolución Industrial, que tuvo lugar a principios del siglo XVIII. Los inventos de esa época dieron origen a una empresa formal conocida como fábrica. Con la proliferación de las fábricas creció la necesidad de coordinar los esfuerzos de grandes cantidades de personas en la producción continua de productos. Esto, a su vez, ocasionó graves problemas como un gran porcentaje de desperdicios, baja productividad y poca eficiencia en los procesos de trabajo de las fábricas.

La problemática provocada por la Revolución Industrial y el reto que impuso la creación desordenada de fábricas provocó que los científicos de la época experimentaran con el manejo de las 
instalaciones para crear procesos más eficientes de trabajo. Frederick Taylor surgió como el padre de la administración científica y estableció que la empresa y la administración deben analizarse de forma científica, y no empírica: la planificación debe sustituir la improvisación.

\section{La Administración en la actualidad}

Al llegar la época contemporánea, proliferan organizaciones de trabajo dentro de un contexto que brinda una enorme variedad de profesionales. Los últimos años han estado acompañados por estudios en búsqueda de estructuras, sistemas y mecanismos orientados a garantizar el mejor funcionamiento de las organizaciones. Esto se hace mediante el uso de estrategias que permitan lograr la colaboración de los sujetos hacia el alcance de fines colectivos.

Dentro de esta perspectiva han surgido un sinnúmero de disciplinas altamente interrelacionadas, como la psicología industrial organizacional, la psicología social del trabajo, el comportamiento organizacional y la gerencia de los recursos humanos. Todas estas disciplinas intentan alcanzar una mejor comprensión de la problemática organizacional enfatizando el aspecto humano y la necesidad de considerar la integración de éste con los elementos técnicos y mecánicos de la producción (Toro, 2002). En efecto, se siguen haciendo esfuerzos con el fin de producir conocimientos que permitan mayores grados de eficacia y productividad. En esta lucha se destacan los factores psicosociales de los sujetos, las relaciones que se establecen entre sus pares y la integración de estos a los factores técnicos y mecánicos. El énfasis en los sujetos implica una inquietud hacia el mejoramiento en la calidad de vida laboral de los trabajadores y consecuentemente en el desarrollo social de las comunidades dentro de las cuales operan las organizaciones (Toro, 2002).

La psicología industrial organizacional es, dentro de esas disciplinas mencionadas, un campo tanto de investigación científica como de práctica profesional, que ayuda a mejorar el bienestar de las personas de diferentes maneras: el entendimiento de la conducta de los individuos y las organizaciones en el lugar de trabajo; las ayudas significativas a los ingresos de las personas, y al enriquecimiento del trabajo; y la ayuda a las organizaciones en la administración efectiva de sus recursos humanos (Kline, 1996). Muchinsky (2005), uno de los teóricos más respetados en el área de psicología industrial organizacional, cita a Guion (1965) al definir la psicología industrial organizacional como "el estudio científico de las relaciones entre el hombre y el mundo del trabajo: el estudio del ajuste que hace la persona en los lugares que va, con la gente que se reúne, y las cosas que hace en el proceso de vivir". Blum y Taylor (en Muchinsky, 2005), definen esta psicología como "aplicación o extensión de hechos y principios psicológicos a los problemas relacionados con los seres humanos dentro del contexto de los negocios o la industria".

Los psicólogos industriales organizacionales, según Kline (1996), deben ser capaces de evaluar críticamente, conducir y aplicar investigaciones en su campo. Estas destrezas son desarrolladas en el trabajo en curso, en las investigaciones de tesis y disertaciones y en experiencias en el campo. Las destrezas incluyen: áreas específicas de la psicología industrial; áreas generales de bases sociales, cognitivas y biológicas del comportamiento; medición, diseño de investigación y análisis 
estadístico; y estándares éticos y práctica profesional.

\section{El comportamiento del ser humano y el ambiente organizacional}

Una de las áreas específicas de interés, relacionadas con el comportamiento organizacional, la gerencia de recursos humanos, la psicología industrial organizacional y el comportamiento humano en general dentro de la organización, es lo que se conoce como ambiente organizacional. Este es el ambiente interno que existe entre los miembros de la organización, el cual está estrechamente ligado al grado de motivación de los empleados (Chiavenato, 2000) y a sus niveles de satisfacción en el trabajo. Cuando estos tienen una gran motivación, el clima motivacional permite establecer relaciones satisfactorias de interés y colaboración. Cuando la motivación es escasa, ya sea por frustración o impedimentos en la satisfacción de necesidades, el clima organizacional tiende a enfriarse y sobreviven estados de depresión, desinterés, apatía descontento hasta llegar a estados de agresividad, agitación e inconformidad, entre otros. Esto podría llevar a situaciones más fuertes, como las huelgas, donde los empleados enfrentan abiertamente a la empresa (Chiavenato, 2000).

James y James (1989), citados por Chiavenato, analizan el ambiente organizacional desde varias dimensiones. Para ellos este ambiente organizacional se constituye como un contexto regulador y facilitador de realidades psicológicas tales como la motivación individual, la satisfacción y el compromiso de los sujetos con sus trabajos. Establecen que en la dimensión individual se desprende el concepto de motivación y en la dimensión organizacional se desprende el concepto de clima organizacional, siendo este un aspecto fundamental en el cual ocurren las relaciones entre los sujetos y las organizaciones.

Las personas en el trabajo se encuentran en un proceso continuo de adaptación a una variedad de situaciones con el fin de satisfacer sus necesidades y mantener el equilibrio individual. Esta adaptación va más allá de una adaptación fisiológica y de seguridad; también incluye la satisfacción de las necesidades sociales y personales, así como las de autoestima y auto realización. Sin embargo, se considera que la satisfacción de las necesidades superiores depende de las oportunidades de interacción con las otras personas, en especial, con las que ocupan puestos de autoridad. La adaptación varía de acuerdo con la particularidad de cada sujeto así como del contexto socio temporal en que se encuentra. También puede variar en grados y fluctuar desde una adaptación excelente, que corresponde a una buena salud mental, hasta una mínima (Chiavenato 2000).

Burke (1994), por otro lado, define el ambiente organizacional como uno constituido por las impresiones de corrientes colectivas, así como por las expectativas y los sentimientos de los miembros de la unidad local de trabajo. Estos afectan las relaciones entre los miembros y supervisores y entre distintas unidades. Para este autor, el concepto de ambiente organizacional emerge como resultado de estudios acerca de los estados psicológicos de los sujetos y los cuales son afectados por las condiciones organizacionales que se han establecido, tanto en sus sistemas como en sus estructuras. Por lo tanto, el clima organizacional representa una dimensión que se puede cambiar. La interacción entre los sujetos y la organización (y como consecuencia el clima organizacional dentro 
de la institución) puede explicarse como un proceso de reciprocidad basado en un contracto psicológico que se establece a partir de las expectativas mutuas que rigen las relaciones de intercambio entre los sujetos y las organizaciones. En ese contrato, las organizaciones ofrecen incentivos y los sujetos, por su parte, contribuyen con su trabajo. El equilibrio dependerá del intercambio entre los incentivos ofrecidos y las contribuciones que se entregan como producto a la organización (Burke, 1994).

Enebral (2007), por otro lado, establece que todas las personas consideran imperante el crecimiento personal y la consecución del bienestar y la felicidad, aunque quizá no siempre se tenga éxito en este empeño. El autor establece que en el ejercicio profesional, uno puede alcanzar mayor grado de desarrollo y de satisfacción; y que si nos preguntáramos dónde disfrutamos más, si en el trabajo o en el ocio, quizá la mayoría diría que en el ocio. Pero al pensarlo mejor admitiríamos que depende. De hecho, hay muchos adictos al trabajo, como los hay a las drogas o al sexo. Aunque en algunos trabajos como el de los bomberos y los controladores aéreos cueste imaginar el disfrute, hay otros, donde queda espacio para disfrutar. En este análisis de la felicidad en el trabajo debemos pensar no solo en la tarea sino también en las circunstancias que la rodean, ya que esa felicidad dependerá de que el reto sea adecuado: ni tan bajo como para aburrirnos, ni tan alto como para producirnos ansiedad.

En esto del ambiente organizacional algunos autores sugieren que, aunque intrínsecamente el trabajo permite realizarnos y aun disfrutar, nos podemos ver afectados por la anomia donde se pueden sumergir las organizaciones en evolución cultural. Esto crea interferencias y circunstancias adversas como excesos burocráticos, condiciones ambientales inadecuadas, agravios comparativos, relaciones poco amistosas, falta de información, clima de temor, politiqueo, neurosis organizacional, desacuerdos funcionales, sofocación de la creatividad y de la iniciativa (Enebral, 2007). Ante esta problemática, Enebral está de acuerdo con lo que McGregor sostenía hace 40 años: los trabajadores están, en su inmensa mayoría, dispuestos a comprometerse y contribuir decididamente con los resultados de la organización, pero también desean disfrutar de su trabajo cotidiano y llegar a casa de buen humor.

Otra forma de analizar y entender la conducta organizacional $\mathrm{y}$, por ende, el ambiente organizacional es analizando la misma desde la perspectiva teórica de Bolman \& Deal (2008) quienes establecen que el análisis de esa conducta organizacional se puede realizar desde cuatro diferentes perspectivas o marcos conceptuales que son: el estructural, el humano, el político y el simbólico. Estos marcos, a su vez, visualizan a la organización como una fábrica, una familia, una selva y un templo. Los cuatro marcos son ventanas en el mundo y lentes que permiten tener el mundo enfocado.

El reto actual, según Bolman \& Deal, en este mundo turbulento y complicado, es encontrar la forma correcta de enmarcar la organización. Desde la perspectiva estructural la organización está guiada por objetivos y un conjunto de políticas establecidas por la alta gerencia. Desde la perspectiva humana se recalca que el mal funcionamiento de la institución surge de las malas relaciones interpersonales y de las dinámicas de grupo. El marco político, por su parte, visualiza a la institución con arenas políticas vivas y turbulentas que albergan 
una red compleja de intereses individuales y grupales. Por último, el marco simbólico visualiza la vida de una forma más fluida que lineal; a las organizaciones como complejas y continuamente cambiantes, máquinas de pinball.

Dentro de esos cuatro marcos, el político sostiene que cuando la organización se enfrenta a diferencias permanentes y recursos escasos, los conflictos son inevitables y el poder es el recurso clave. El concepto de recursos escasos sugiere que la política será más intensa y sobresaliente en los tiempos difíciles. Por otro lado, el poder, que es la otra clave en el marco político, es la capacidad de lograr que las cosas pasen. Por último, el marco político recalca, que los objetivos organizacionales son establecidos mediante los procesos de negociación en interacción entre los jugadores claves y no por los niveles altos.

Ian C. Mac Millan (1980), por su parte, señala en su libro Strategic Formulation: Political Concepts que el comportamiento político que necesita ser estudiado es la formación de coaliciones. Él visualiza la conducta del individuo dentro de las organizaciones en términos de su propio interés hacia los otros miembros o partes de la institución. En el comportamiento político dentro de las instituciones, las personas realizan dos tipos de actividades políticas, unas de manipulación y otras de acomodo. En ese proceso, si las personas se dan cuenta que las acciones de los demás pueden afectar sus metas, entonces podrían hacer pequeños ajustes. Por último, Mac Millan señala que los resultados de las acciones políticas determinan el papel de las personas en sus acciones sociales dentro de los diferentes grupos en los que está afiliado.

Pfeffer, que es otro teórico en el área de psicología industrial organizacional, define poder como la habilidad potencial de influenciar el comportamiento, cambiar el curso de los eventos, enfrentar la resistencia y lograr que las personas hagan cosas que ellos en otras circunstancias no harían. Siguiendo el análisis de poder, Peffer indica que el diagnóstico del poder dentro de la organización es muy importante por varias razones. En primer lugar, el ejercicio y el uso del poder es facilitado por un diagnóstico preciso de las situaciones políticas que enfrentan los diferentes actores sociales. Segundo, la medición y el avalúo del poder es importante para aquellos que investigan el tema. Tercero, una forma de entender el poder es considerar cómo los conceptos son o pueden ser examinados y usados.

\section{Conclusiones}

La psicología industrial organizacional se enfrenta en la actualidad a una tarea muy difícil: aumentar la armonía o adaptación entre la fuerza de trabajo y el lugar de trabajo, en una época cuando la composición de ambos está cambiando rápidamente. La fuerza obrera actual es diferente de otras épocas: más personas buscan trabajo; empleados con un nivel superior de educación; más mujeres en la fuerza laboral; más trabajo en la industria del servicio; los trabajos requieren conocimientos en el manejo de las computadoras; un gran número de personas trabajando a tiempo parcial. Los cambios económicos, por otro lado, han forzado a despidos en masa, lo que lleva en ocasiones a que las personas tengan que aprender nuevas destrezas. Los cambios sociales también están afectando el empleo en términos generales ya que llevan nuevos problemas al lugar de trabajo, por ejemplo, los relacionados con el consumo de drogas (Muchinsky, 2005). 
En el caso de Puerto Rico, los cambios en la estructura ocupacional e industrial del empleo han sido transformados gradualmente por factores económicos, políticos, sociales, familiares, culturales, migratorios, movilidad laboral y falta de planificación de los recursos humanos, entre otros (Rivera Aponte y otros, 2007). Las modificaciones generadas en la economía local a partir de la década de 1960 propiciaron cambios sociales significativos que alteraron la vida de nuestra población (Junta de Planificación, 1982). En el comienzo del siglo XXI, por ejemplo ya las nuevas corrientes de pensamiento ubican a la mujer en una posición de franca igualdad con el hombre respecto al disfrute de accesos, derechos, equidades, oportunidades y justicias sociales. Las corrientes feministas han generado conciencia en la lucha por la equidad, el acceso y la participación del género femenino en la fuerza laboral puertorriqueña.

Después de revisar la literatura relacionada a actitudes y satisfacción en el trabajo, y considerando el trabajo de varios estudiosos del área, se concluye que en una larga historia de estudios de satisfacción en el trabajo, no se ha encontrado una regla general sobre cómo las diferentes facetas de la satisfacción en el trabajo forman una satisfacción general (Alas, 2005). Los cambios en el ambiente laboral han llevado a cambios en la naturaleza del trabajo, que han influenciado la forma como las actitudes en el trabajo son estudiadas; además, el papel desempeñado por el ambiente social no ha sido bien enfatizado en el estudio de esas actitudes. Por lo tanto, es necesario realizar estudios adicionales para descubrir cuáles facetas del constructo de satisfacción en el trabajo son más importantes para la satisfacción general del trabajo y cómo el ambiente social ha influenciado en esas facetas (Alas, 2005). Esto aplica especialmente al Puerto Rico actual que está viviendo una grave crisis económica que ocasiona insatisfacción en el trabajo (Rodríguez, 2006) e inestabilidad económica y emocional, tanto a empleados públicos como privados, que trabajan en un clima laboral inestable; donde se ven o se pueden ver amenazados por la posibilidad de perder beneficios actuales, del retiro y hasta sus empleos.

\section{Referencias}

Álvarez, Zancudo Rivas (1992). El constructo clima organizacional: concepto teorías, investigaciones y resultados relevantes. Revista Interamericana de Psicología Ocupacional, 11(1-2), 25-50.

Argyris, C. y Schon, D. (1996). Organizational Learning II: Theory, Method and Practice. Reading, MA: Addison-Wesley Publishing Company.

Aronowitz, S. y DiFazio,W. (1995). Jobless Futures. Minessota, Estados Unidos: University of Minessota Press.

Ballina Ríos, F. (2000). Teoría de la administración: un enfoque alternativo, México: McGRAWHILL Interamericana Editores, S.A. de C.V.

Bolman, L. y Deal, T. (2008). Reframing Organization (3ra edición). San Francisco: Jossey- Bass Publishers.

Burke, R. J., \& Cooper, C. L. (2004). Leading in Turbulent Times: Management in the New World of Work, Blackwell Publishing.

Burke, W.W., (1994). Organizational Development. A Process of Learning and Changing Reading, MA: Addison-Wesley Publishing Company.

Castelló, A. (2002). Asunciones y creencias que están en juego, y que, en todo caso, deben ser objeto de análisis e intervención, Psicothema. , 15 (3), 362-368.

Chiavenato, I. (2000). Administración de recursos humanos. Colombia: McGraw Hill. (5Ed.) 
Cirino Gerena, G. (2000). Pasado, presente y futuro de la psicología industrial organizacional en Puerto Rico. R. interam. Psicol. 34(2), 119-126

El trabajo en el ocaso de las carreras. $(2007,11,20)$. Recuperado de www. gestiopolis.com/recursos/ documentos/fulldocs/rrhh/ocaso.htm.

Enebral Fernández, J. (2007,11) Clima organizacional y satisfacción en el trabajo: En busca de la satisfacción y aun disfrute, en el desempeño profesional. Recuperado de www.arearh.com/rrhh/satisfaccion.htm.

French, W. L. y Bell, C. H. (2002). Organizational Development: Behavior Science Interventions for Organizational Improvement. Estados Unidos: Kindle Edition.

Frederick Herzberg's Motivation and Hygiene Factors. Recuperado de http://www .businessball.com/herzber.htm

Galbraigh, J. (1977). Designing Complex Organizations, San Juan: Fondo Educativo Interamericano.

Gergen, K.J. (1993). Organization Theory in the Postmodern Era. Rethinking Organization: New Directions in Organization Theory and Analysis. Londres: Sage Publications, Inc.

Gibson, Ivancevich y Donnely (1997). Las organizaciones, ED. Mc Graw Hill.

Greenberg, J. y Baron, R. A. (2003). (Octava edición), Behavior in Organizations. New Jersey, E. U: Prentice Hall.

Hackman, R., Oldham, g. y Purdy, K. (1994). . In Gold, B.A, (Ed.). Job Design: A New Strategy for Job Enrichment. New Cork: Dryden Press.

Hair, Jr., J. F., Bush, R. P. y Ortinau, D. J. (2004) Investigación de mercados. México: McGraw Hill Interamerica Editores, S. A.

Hellriegel, D., Slocum, Jr., J.W. y Woodman, R.W. (1998). (Octava Edición), Comportamiento organizacional, International Thomson Editores.

Herzberg, F., Mausner, B., \& Snyderman, B. B. (1959). (2nd Ed.). The Motivation to Work New York: John Wiley \& Sons.
Herzberg, F. (1987). Workers' Needs. Industry Week, 234 (6), 29.

Herzberg's Motivation-Hygiene Theory .Changing Minds. (2008). Recuperado de http: //changinfminds.org/explanations/needs/herz berg_needs.htm.

Hodge, A. , Anthony, W.P. (2002). (Sexta Edicion), Organization Theory: A Strategic Approach. Prentice Hall

Jackson, Susan E. \& Schuler, Randall S. (2000) Management Human Resources: A Partnership Perspective. Estados Unidos: South-Western College Publishing.

Johns, G. (1998). The Nature of Work. The Context of Organizational Behavior, and the Application of Industrial-Organizational Psychology. Canadian Psychology, 39, 149.

'Katzell, R. y Austin, J.T. (1992). From Them to Now: The Development of Industrial Organizational Psychology in the United States. Journal of Applied Psychology, 77 (6), 803-829.

Kline, T. (1996). Defining the Field of IndustrialOrganizational Psychology. Canadian Psychology, 37, 205.

Lewin, K. (1948). Gertrude W. Lewin (Ed.). Resolving Social Conflicts; Selected Papers on Group Dynamics. New York: Harper \& Row.

Mac Millan, Ian C (1980). Strategic Formulation: Political Concepts. The West series in Business Policy and Planning. The Author

Martinez Ibarra, E.A, (2009). Actitudes y Satisfacción hacia el trabajo de los profesores de la Facultad de Administración de Empresas de Recinto de Rio Piedras de la Universidad de Puerto Rico, Universidad de Puerto Rico, San Juan, Puerto Rico. Disponible en biblioteca.urrp.edu/LNA/LNA\%20abriljunio\%2011.htm

Muchinsky, P.M. (1993). Psychology Applied to Work. Estados Unidos: Kindle Edition.

Pfeffer, J. (1981). Power in Organizations. Marshfield, Mass: Pitman Publish. 
Rivera Aponte. A., Ruiz Mercado A.L. y García Toro, V.I. (2007). Mujer y brecha salarial: Reto del Siglo XXI. Puerto Rico: Oficina de la procuradora de las mujeres.

Rodríguez, Villegas, J. J. (2006, 11 de diciebre). Revela un sondeo la satisfacción de boricuas con sus jefes y trabajos, El Nuevo Día, pp.49.

Sari, L. M. y Judge, T.A. (2004). Saari, L. M. \& Judge, T. A. (2004). Employee Attitudes and Job Satisfaction. Human Resource Management, 43, 395-407

Toro Díaz, C. A. (2002). Los errores de diseño y el aprendizaje en las organizaciones: teoría, evidencia empírica y aplicaciones prácticas. San Juan: Publicaciones Puertorriqueñas.

Zillman, M. (2000). Motivation, Organizational Behavior. Recuperado de http:// academic emporia.edu/smithwil/00fallmg443/eja/zillm an.html.

\section{Información de la autora}

La Dra. Elena A. Martínez Ibarra es catedrática de la Universidad de Puerto Rico. Tiene una maestría en Mercadeo, estudios doctorales en Comercio Internacional y un doctorado en Psicología Industrial Organizacional. La profesora es consultora de mercadeo y de Psicología Industrial Organizacional y tiene amplia experiencia en el desarrollo de estudios de mercado, conducta del consumidor, satisfacción en el trabajo y estudios políticos, entre otros. 